\title{
RADIOCARBON DATING SITES OF NORTHWEST RUSSIA AND LATVIA
}

\author{
G. I. ZAITSEVA and S. G. POPOV
}

Radiocarbon Laboratory, Institute of the History of Material Culture, Russian Academy of Sciences, Dvortsovaya Naberezhnaya 18, St. Petersburg 191186 Russia

\begin{abstract}
We describe applications of radiocarbon dating used for establishing a chronology of archaeological sites of the Novgorod region at the end of the first millennium $\mathrm{AD}$. We have ${ }^{14} \mathrm{C}$-dated known-age tree rings from sites in Latvia and ancient Novgorod, northwest Russia, as well as charcoal and wood from Novgorod. Calendar ages of ${ }^{14} \mathrm{C}$-dated tree rings span the interval, AD 765-999. We used the Groningen calibration program, CAL15 (van der Plicht 1993) to calibrate ${ }^{14} \mathrm{C}$ ages to calendar years. Comparisons between ${ }^{14} \mathrm{C}$ results and archaeological data show good agreement, and enable us to narrow the calendar interval of calibrated ${ }^{14} \mathrm{C}$ determinations.
\end{abstract}

\section{INTRODUCTION}

Systematic excavations of old cities in northwest Russia, mainly Novgorod, have led to the construction of an archaeological chronology of the 10th-15th centuries. Lesman $(1984,1990)$ has linked Russian burial sites in northwest Russia with the well-dated dendrochronological scale of ancient Novgorod. This enables us to place the origin of many burial complexes at the beginning to the middle of the second millennium $\mathrm{AD}$.

Pre-Christian Slavic settlement of northwest Russia has attracted much attention since the end of the 19th century. Recently excavated material from related settlements supplements the information retrieved from burial sites of long-barrow and high mound (sopki) cultures. However, chronological data are scarce (Popov, Svezhentsev and Zaitseva 1993).

The ancient city of Novgorod provides a well-documented archaeological chronology for northwest Russia. Dendrochronologically dated wood samples (Chernykh 1985) and the reconstruction of pavement-level stratigraphy at the Troitskii-VIII site (Bassalygo, Sorokin and Khoroshev 1988) indicate that the settlement at Novgorod extends from the mid-9th to the early 15 th centuries. Our research on ${ }^{14} \mathrm{C}$-dating tree rings has narrowed the period of occupation to $\mathrm{AD} 765-1000$. Figure 1 shows the archaeological sites of the Novgorod region.

\section{METHODS}

We used dendrochronologically dated pine wood samples for ${ }^{14} \mathrm{C}$-dating 14 samples from the sites of Ushuri and Araishiu, Latvia (which belong to the western European forest zone and are synchronous with some layers of ancient Novgorod), and 25 samples from the Troitskii-VIII excavation in Novgorod (Chernykh 1985a,b, 1987; Urieva 1989).

Wood samples were pretreated by using benzene/alcohol 2:1 for resin removal and acid-alkali-acid (AAA) solutions. The sequence follows: extraction of resins for $5-6 \mathrm{~h} ; 1 \% \mathrm{HCl}$ solution at room temperature for $1-2 \mathrm{~h} ; 0.5 \% \mathrm{NaOH}$ solution at $80^{\circ} \mathrm{C}$ for $1 \mathrm{~h}$; washing with hot water; $1 \% \mathrm{HCl}$ solution at $80^{\circ} \mathrm{C}$ for $1 \mathrm{~h}$; and finally, rinsing with hot water to $\mathrm{pH}$ 7. The yield of this procedure is $c a$. $60-70 \%$ by weight. The samples were then carbonized by the dry distillation method (anoxic). We reacted the carbonized samples with lithium, then used standard procedures to convert $\mathrm{Li}_{2} \mathrm{C}_{2}$ to $\mathrm{C}_{6} \mathrm{H}_{6}$. The ${ }^{14} \mathrm{C}$ activity was measured using liquid scintillation spectrometry. We used a two-channel analyzer with quartz vials of $3.2-$ and 6.8 - $\mathrm{ml}$ capacities. We did not correct for isotopic fractionation. The ratio of our calibration standard to the international standard, SRM-4990 (Arslanov 


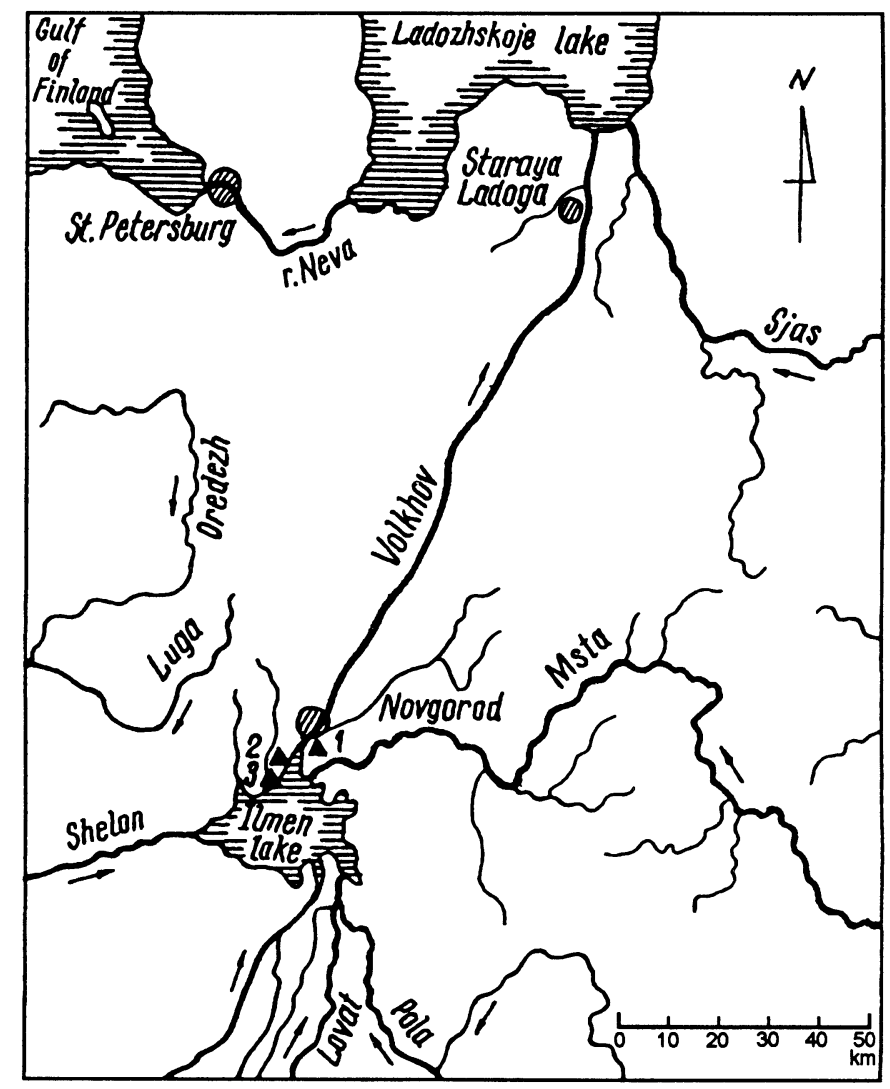

Fig. 1. Map of the Novgorod region and archaeological sites mentioned in the text: 1. Ryurikovo Gorodishche; 2. Gorodishche Georgii; 3. Vasiljevskoe-I settlement

1987 ) was $4.993 \pm 0.011$. The statistical error depended on the number of counts accumulated. Generally, the $1 \sigma$ error was between 30 and $60 \mathrm{yr}$, sometimes 70 and $90 \mathrm{yr}$.

\section{RESULTS}

Earlier excavations yielded ${ }^{14} \mathrm{C}$ data sets for dendrochronologically dated wood samples from the 25th level of the Troitskii-VIII site in ancient Novgorod: TC-VIII-25-78, felling date: AD 968; and TC-VIII-25-63, felling date: AD 960 (Table 1, Fig. 2: III-IV). In the three years since we wrote our last report (Popov, Svezhentsev and Zaitseva 1993), we have obtained more data from Layers 22, 26 and 27 of the same excavation: TC-VIII-22-50-wood from the framework, felling date: AD 1002; TC-VIII-26-88-wood from planking, felling date: AD 960; and TC-VIII-27-130-wood from a wall, felling date: AD 958 (Table 1, Fig. 2: V-VII). Further, we have obtained dating results of dendrochronologically dated wood from Latvia: Ushuri-wood from planking, felling date: AD846; and Araishiu—wood from planking, felling date: AD 920-930 (Table 1, Fig. 2: I, II)

We have also reconsidered previous conclusions (Popov, Svezhentsev and Zaitseva 1993) based on new results and recent calibration information (Stuiver and Pearson 1993). We used the calibration program CAL15 (van der Plicht 1993) to convert ${ }^{14} \mathrm{C}$ determinations to calendar ages. We report 


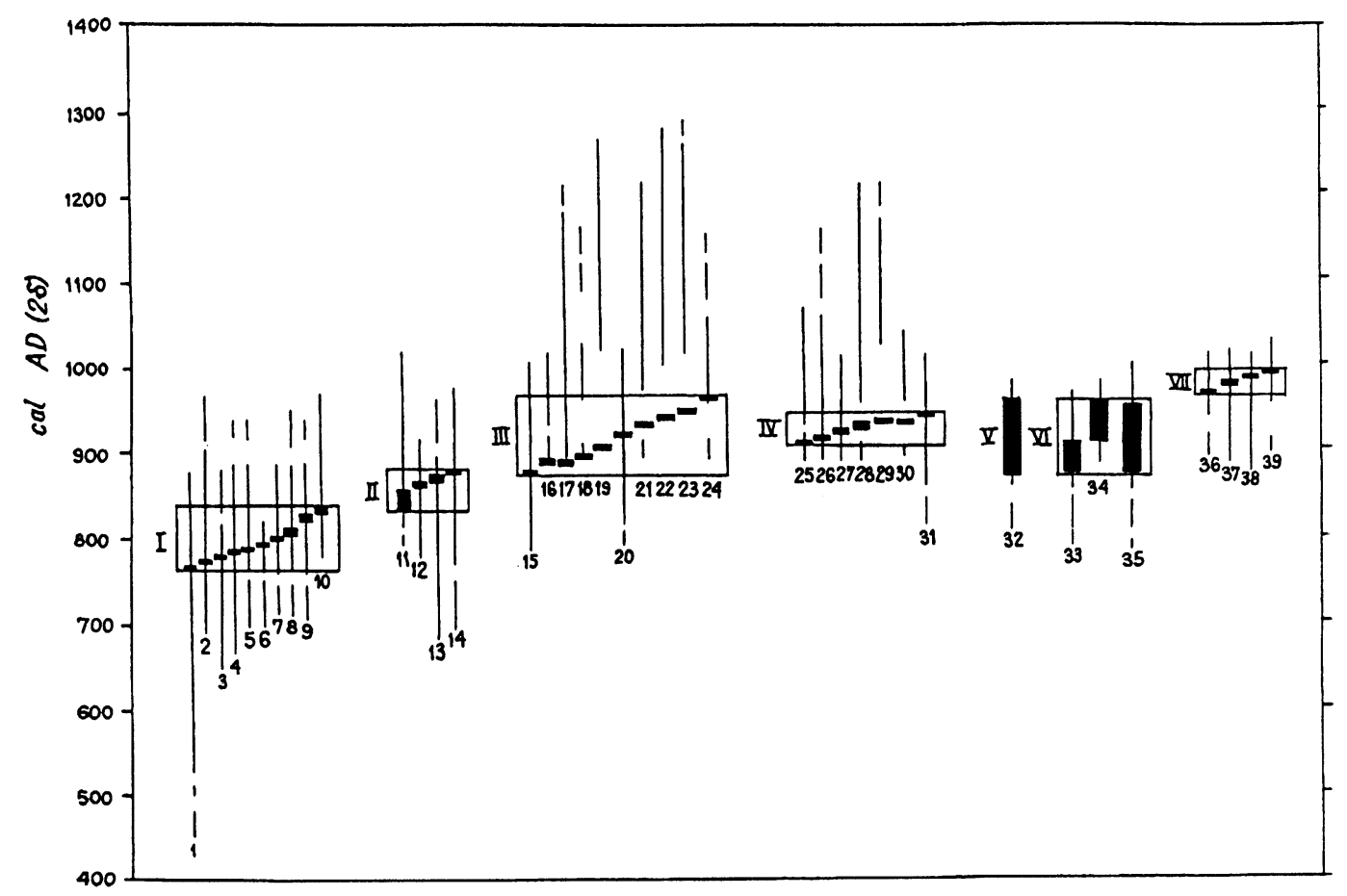

Fig. 2. Calibrated ${ }^{14} \mathrm{C}$ dates $(2 \sigma)$ of tree-ring-dated wood samples: $\mathrm{I}-\mathrm{VII}=$ blocks of tree-ring-dated wood samples; range of tree-ring dates; Nos. 1-39 = sample numbers correlated to Table 1.

our results in Table 1 and in Figures 2 and 3. Figure 3 shows a rapid decline in ${ }^{14} \mathrm{C}$ concentration in Section 3 of the curve at $c a$. cal AD 900 . Some samples of dendrochronologically dated wood lie within this period.

Table 1 compares calendar ages for wood (determined dendrochronologically) with calibrated ${ }^{14} \mathrm{C}$ ages (Fig. 2: Blocks I-VII). Generally, the two data sets agree, particularly where the ${ }^{14} \mathrm{C}$ concentration changes smoothly (Fig. 3: 1, 2, 4). Blocks I, II and IV (Fig. 2) correspond to these sections

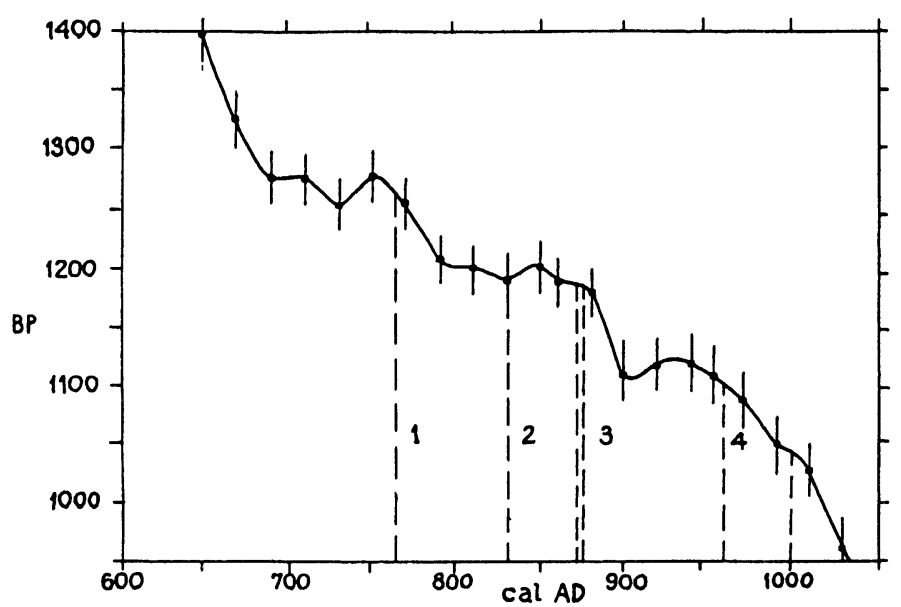

Fig. 3. The Stuiver and Pearson (1993) calibration curve for $\mathrm{AD} 600-1050$ according to the range of ${ }^{14} \mathrm{C}$-dated tree-ring dates 
and tree-ring dates lie inside the calibrated range of ${ }^{14} \mathrm{C}$ ages. Blocks III and IV show some differences between calibrated and tree-ring dates, which may be connected with the section of the calibration curve (Fig. 3: 3), where the ${ }^{14} \mathrm{C}$ concentration quickly changes. It is important to note that the tree-ring samples cover 1-4 rings and have been dated on a bidecadal scale. Measurement may reflect the fine structure of the curve at this point. One cannot fully exclude poorer precision of measurement, on the one hand, and errors in determining tree-ring ages, on the other. In all cases, uncertainties exist in calibrating ages of tree-ring samples; calendar ranges are sometimes wider than tree-ring dates. However, these uncertainties can be smoothed by dating large quantities of tree rings, as Blocks V and VI (Fig. 2) illustrate. We dated Block V as a whole (AD 873-960); we divided VI into 3 samples: the 1st included 35 inner tree rings; the 2nd, 45 external tree rings; and the 3rd included tree rings from AD 879-958.

\section{DATING ARCHAEOLOGICAL SITES OF THE NOVGOROD REGION}

Figure 1 shows locations of archaeological sites of the Novgorod region reported here. Of primary importance in studying ancient Novgorod are the complexes of Ryurikovo Gorodishche, the tradecraft and military-administrative center preceding Novgorod. Ryurikovo Gorodishche is the earliest fortified site at the source of the Volkhov River, dating to the 9th century AD (Nosov 1990). The excavation of a moat in 1987-1989 from a depth of $4.5 \mathrm{~m}$ from the ancient surface confirmed the presence of the fortification, in contrast to the opinion of some archeologists (Lebedev 1985). Archaeologically, the moat dates to the 9-10th centuries. Table 2 and Figures 4 and 5 show the ${ }^{14} \mathrm{C}$ data sets and corresponding calendar ranges for 1 and $2 \sigma$, respectively. Results obtained on charcoal samples from different levels date the moat to the 7 th to the 10 th centuries. The moat may have been built between the early 7 th and 8th century (LE-3467, 3469). LE-3332 suggests occupation of the site to the Early Iron Age, which also concurs with the presence of pottery typical for this time. The moat was filled in at the time the city was replanned in the 11th century AD (Nosov 1990). A charcoal sample, LE-3333, from a lime-firing kiln, agrees well on stratigraphic, archaeologic and ${ }^{14} \mathrm{C}$ grounds. Erected on the site of the filled-in moat, the kiln is strongly linked to the construction of Blagoveschenje Church in AD 1103 (Nosov 1990). One of the calibrated ranges subsumes this date.

The rich wood buildings with galleries discovered at Ryurikovo Gorodishche are associated with a later period. These buildings must have belonged to royalty (Nosov 1990), as their foundations were dug into the fill of a moat. Artifactual and ceramic assemblages date to the 12th-14th centuries. Three ${ }^{14} \mathrm{C}$ dates (LE-4405, -4411, -3935) from the remains of logs and charcoal at the base of the complex date initial construction to the second half of the 13th century. Six samples ( 5 charcoal and 1 wood: LE-4406 to $-4408 \mathrm{a},-4412,-4414)$ date the destruction of the complexes by fire to the second half of the 15th century.

In the Novgorod area, 34 settlements with cultural layers from the end of the first millennium $A D$ are known from the Ilmen Lake district (Poozerje) and from the upper Volkhov River. Samples from two of these sites, Vasiljevskoe-I and Georgii, on the Veryazha River, yielded two representative ${ }^{14} \mathrm{C}$ data sets. According to the archaeological remains, Vasiljevskoe-I dates fall between the 9th and 10th centuries (Nosov 1990). The calibrated dates (LE-4157, -4388 to -4392 and -3327 to -3329) on charcoal determine the duration of occupation from AD 956-1000(1020) (Table 2, Fig. 5). Beads and Ladoga-type pottery found at the excavation confirm these dates. However, the possibility of earlier habitation cannot be ruled out (Table 2, Fig. 5).

The site of Gorodishche Georgii, situated $0.3 \mathrm{~km}$ upstream on the Veryazha River, was dated archaeologically to the 8th-9th centuries (Orlov and Aksenov 1961). Recent excavations (Nosov 1990) 
established settlement during the 9th century. Samples LE-3460, and -3937 to -3943 , date part of the settlement to $c a$. AD 925-999. Charcoal from Squares 2 and 3 yielded a calibrated range from the end of the 8th to the 9th centuries (LE-3461). Two calibrated dates on charcoal from fortified buildings (LE-3934, -3935) support this interval, but cannot exclude later occupation in the 9th10th centuries. Archaeological evidence (Nosov 1990) corroborates the synchronic existence of Vasiljevskoe-I and Gorodishche Georgii, at least at one point (LE-3936), during the Early Iron Age.

Historically associated with ancient Novgorod is the famous site of northwest Russia, Staraya Ladoga (Zemlyanoe Gorodishche), which was first excavated in 1909 (Kirpichnikov 1985). Chernykh (1987) combined dendrochronological time scales of Staraya Ladoga with ancient Novgorod. We tree-ring dated two wood samples from Layer E3 (AD 760-830) (Chernykh 1985a). The sample without a tree-ring date was divided into two (1 of sapwood and 1 of heartwood). The calibrated range for samples LE-4158 and -4159 is the same as the dendrochronological period of Layer E3.

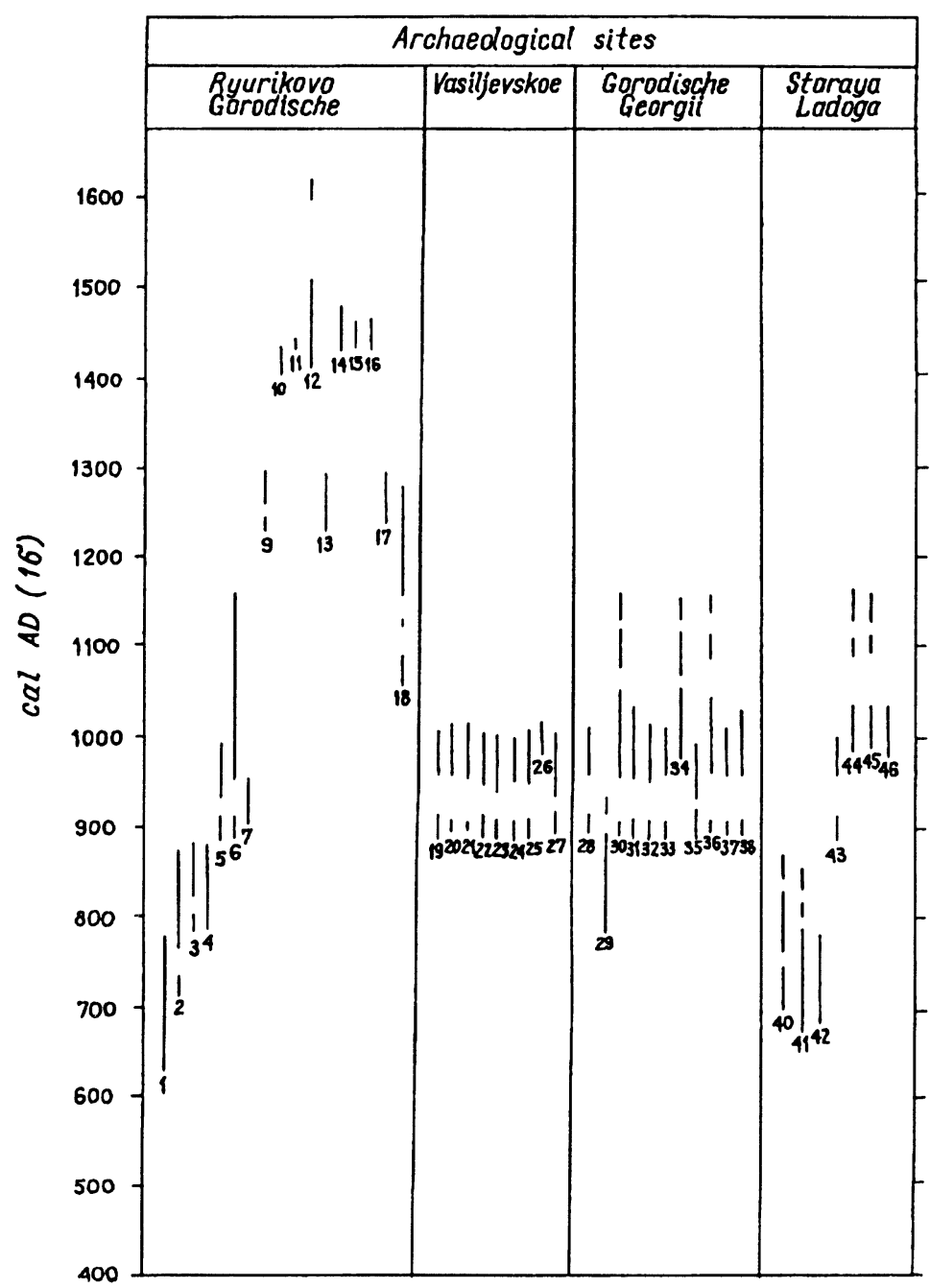

Fig. 4. Calibrated ${ }^{14} \mathrm{C}$ dates $(1 \sigma)$ on charcoal and wood samples for the Novgorod region. Nos. $1-46=$ sample numbers correlated with Table 2 . 
An oak sample containing 27 rings from a building erected in AD 776-811 (Chernykh 1989) yielded a calibrated ${ }^{14} \mathrm{C}$ date (LE-4795) of $\mathrm{AD} 680-786$, which lies within the dendrochronological range. ${ }^{14} \mathrm{C}$ ages of planks and logs (LE-4416 to -4419) agree with archaeological ages, and date to the second half of the 10th century.

\section{ConCLuSION}

${ }^{14} \mathrm{C}$ dating of archaeological sites of the Novgorod region is effective for time scales requiring highprecision dating. Shorter calendar intervals can be obtained only by serial dating and comparisons with results obtained using other dating methods. Archaeological sites of the Novgorod region of the end of the first millennium $\mathrm{AD}$ allow us to compare the ${ }^{14} \mathrm{C}$ data with dendrochronological, archaeological and historical documentation. ${ }^{14} \mathrm{C}$ dating of dendrochronologically dated tree rings demonstrated that uncertainties can result from converting ${ }^{14} \mathrm{C}$ years to calendar years. We have

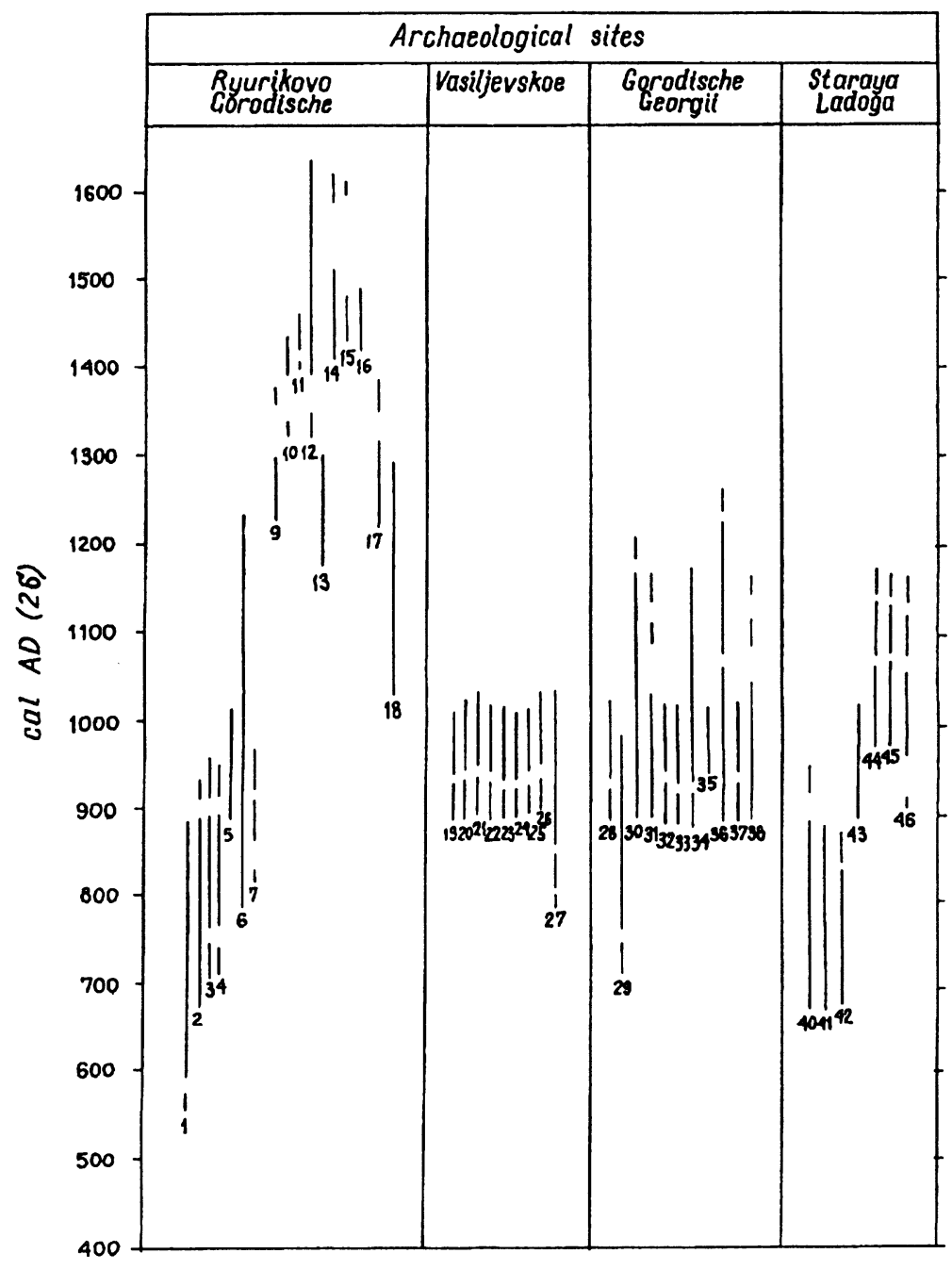

Fig. 5. Calibrated ${ }^{14} \mathrm{C}$ dates $(2 \sigma)$ for the Novgorod region. For key, see Fig. 4. 
found that at least 10-20 tree rings are needed to obtain reliable dates and to show close agreement between calibrated ${ }^{14} \mathrm{C}$ ranges and tree-ring dates.

The ${ }^{14} \mathrm{C}$ data set for archaeological sites of the Novgorod region and Staraya Ladoga is important for studying Slavic history. Staraya Ladoga was the oldest settlement, dating to the 7th-8th century $\mathrm{AD}$; the lower layers of Ryurikovo Gorodishche are also associated with the same period. The sites of Vasilijevskoe-I and Georgii co-existed during the 9th-10th century AD. Future research for this area should link the history of northwestern Russia to the chronology of ancient Novgorod.

\section{ACKNOWLEDGMENTS}

We thank N. B. Chernykh and A. F. Urjeva for providing tree-ring-dated wood samples and E. N. Nosov for analyzing archaeological samples; we also appreciate their discussions of Novgorod archaeology.

TABLE 1. Radiocarbon Dates of Tree-Ring-Dated Wood

\begin{tabular}{|c|c|c|c|c|c|}
\hline \multirow[b]{2}{*}{ No. } & \multirow[b]{2}{*}{ Lab no. } & \multirow{2}{*}{$\begin{array}{c}\text { Tree-ring } \\
\text { ages } \\
(\mathrm{AD})\end{array}$} & \multirow{2}{*}{$\begin{array}{l}\text { Uncalibrated } \\
{ }^{14} \mathrm{C} \text { date } \\
\text { (BP) }\end{array}$} & \multicolumn{2}{|c|}{$\begin{array}{l}\text { Calibrated range (van der } \\
\text { Plicht 1993) (cal AD) }\end{array}$} \\
\hline & & & & $1 \sigma$ & $2 \sigma$ \\
\hline \multicolumn{6}{|c|}{ Latvia - Ushuri, 1964 Excavation (Block 1) } \\
\hline 1 & LE-4244 & 767 & $1386 \pm 90$ & $\begin{array}{l}564-570 \\
596-726, \\
732-772\end{array}$ & $\begin{array}{l}454-480, \\
540-512, \\
530-880\end{array}$ \\
\hline 2 & LE-4243 & 772 & $1205 \pm 60$ & $\begin{array}{l}724-734 \\
772-892 \\
922-941\end{array}$ & $\begin{array}{l}\text { 690-902, } \\
904-968\end{array}$ \\
\hline 3 & LE-4242 & 777 & $1297 \pm 50$ & $674-772$ & $\begin{array}{l}658-824, \\
836-870\end{array}$ \\
\hline 4 & LE-4241 & 781 & $1253 \pm 60$ & $\begin{array}{l}694-752 \\
758-820 \\
838-866\end{array}$ & $\begin{array}{l}\text { 662-892, } \\
922-942\end{array}$ \\
\hline 5 & LE-4240 & $786-787$ & $1217 \pm 40$ & $782-876$ & $\begin{array}{l}694-752, \\
758-892, \\
920-947\end{array}$ \\
\hline 6 & LE-3632 & $796-798$ & $1235 \pm 35$ & $774-874$ & $\begin{array}{l}694-752 \\
758-884\end{array}$ \\
\hline 7 & LE-4238 & $798-801$ & $1226 \pm 30$ & $\begin{array}{l}780-824 \\
836-872\end{array}$ & $\begin{array}{l}714-742 \\
766-886\end{array}$ \\
\hline 8 & LE-3633 & $804-808$ & $1211 \pm 40$ & $786-878$ & $\begin{array}{l}706-748, \\
762-849, \\
916-957\end{array}$ \\
\hline 9 & LE-3635 & $818-828$ & $1216 \pm 35$ & $\begin{array}{l}786-828 \\
832-874\end{array}$ & $\begin{array}{l}916-957 \\
706-748, \\
762-892, \\
922-940\end{array}$ \\
\hline 10 & LE-3636 & $829-835$ & $1175 \pm 40$ & $\begin{array}{l}792-802 \\
814-846 \\
853-892 \\
918-952\end{array}$ & $780-968$ \\
\hline
\end{tabular}


Table 1. (Continued)

\begin{tabular}{|c|c|c|c|c|c|}
\hline \multirow[b]{2}{*}{ No. } & \multirow[b]{2}{*}{ Lab no. } & \multirow{2}{*}{$\begin{array}{c}\text { Tree-ring } \\
\text { ages } \\
(\mathrm{AD})\end{array}$} & \multirow{2}{*}{$\begin{array}{l}\text { Uncalibrated } \\
{ }^{14} \mathrm{C} \text { date } \\
\text { (BP) }\end{array}$} & \multicolumn{2}{|c|}{$\begin{array}{l}\text { Calibrated range (van der } \\
\text { Plicht 1993) (cal AD) }\end{array}$} \\
\hline & & & & $1 \sigma$ & $2 \sigma$ \\
\hline \multicolumn{6}{|c|}{ Latvia-Araishiu, 1965-1967 Excavation (Block II) } \\
\hline 11 & LE-4225 & $835-855$ & $1109 \pm 55$ & $890-988$ & $\begin{array}{l}792-802 \\
814-848 \\
852-1022\end{array}$ \\
\hline 12 & LE-4224 & $861-865$ & $1182 \pm 40$ & $\begin{array}{l}\text { 788-892, } \\
924-934\end{array}$ & $776-968$ \\
\hline 13 & LE-4223 & $866-872$ & $1210 \pm 60$ & $\begin{array}{l}718-738 \\
770-892 \\
926-934\end{array}$ & $\begin{array}{l}684-900 \\
906-966\end{array}$ \\
\hline 14 & LE-4222 & $872-876$ & $1190 \pm 60$ & $\begin{array}{l}780-892 \\
920-951\end{array}$ & $\begin{array}{l}696-750 \\
758-980\end{array}$ \\
\hline \multicolumn{6}{|c|}{$\begin{array}{l}\text { Ancient Novgorod, Troitskii-VIII, } 1987 \text { Excavation } \\
\text { TC-Y111-25-78 (Block III) }\end{array}$} \\
\hline 15 & LE-4571 & 878 & $1143 \pm 55$ & $\begin{array}{l}824-836 \\
872-984\end{array}$ & 784-1006 \\
\hline 16 & LE-4572 & $882-884$ & $1084 \pm 30$ & $\begin{array}{l}896-910 \\
963-1008\end{array}$ & $\begin{array}{l}892-924 \\
936-1016\end{array}$ \\
\hline 17 & LE-4573 & 888 & $1011 \pm 70$ & $\begin{array}{c}972-1058 \\
1078-1124 \\
1134-1160\end{array}$ & $\begin{array}{r}890-1177, \\
1192-1206\end{array}$ \\
\hline 18 & LE-4574 & $892-895$ & $1022 \pm 35$ & $988-1030$ & $\begin{array}{c}900-906, \\
966-1050, \\
1088-1118, \\
1138-1158\end{array}$ \\
\hline 19 & LE-4576 & $901-902$ & $899 \pm 70$ & $\begin{array}{l}1040-1098 \\
1112-1146\end{array}$ & $1020-1269$ \\
\hline 20 & LE-4580 & $923-924$ & $1104 \pm 60$ & $890-996$ & $\begin{array}{l}790-806 \\
812-1026\end{array}$ \\
\hline 21 & LE-4581 & $930-935$ & $1014 \pm 50$ & $\begin{array}{l}978-1044, \\
1092-1116, \\
1142-1156\end{array}$ & $\begin{array}{l}894-912 \\
957-1164\end{array}$ \\
\hline 22 & LE-4582 & $936-937$ & $919 \pm 80$ & $\begin{array}{l}1032-1177 \\
1190-1208\end{array}$ & $1002-1275$ \\
\hline 23 & LE-4583 & $943-950$ & $907 \pm 70$ & $\begin{array}{l}1040-1100 \\
1110-1146 \\
1152-1212\end{array}$ & $\begin{array}{l}1016-1260 \\
1262-1268\end{array}$ \\
\hline 24 & LE-4586 & $958-962$ & $1028 \pm 40$ & $978-1032$ & $\begin{array}{c}894-916, \\
956-1054, \\
1082-1122, \\
1136-1158\end{array}$ \\
\hline & \multicolumn{5}{|c|}{$T C-Y 111-25-63($ Block IV) } \\
\hline 25 & LE-4589 & $913-916$ & $1018 \pm 60$ & 970-1052, & \\
\hline
\end{tabular}


Table 1. (Continued)

\begin{tabular}{|c|c|c|c|c|c|}
\hline \multirow[b]{2}{*}{ No. } & \multirow[b]{2}{*}{ Lab no. } & \multirow{2}{*}{$\begin{array}{l}\text { Tree-ring } \\
\text { ages } \\
\text { (AD) }\end{array}$} & \multirow{2}{*}{$\begin{array}{l}\text { Uncalibrated } \\
{ }^{14} \mathrm{C} \text { date } \\
\text { (BP) }\end{array}$} & \multicolumn{2}{|c|}{$\begin{array}{l}\text { Calibrated range (van der } \\
\text { Plicht 1993) (cal AD) }\end{array}$} \\
\hline & & & & $1 \sigma$ & $2 \sigma$ \\
\hline 26 & LE-4591 & $921-922$ & $1046 \pm 55$ & $\begin{array}{c}1086-1118 \\
1138-1158 \\
894-918, \\
956-1034\end{array}$ & $\begin{array}{c}892-922, \\
936-1166 \\
886-1056 \\
1082-1122, \\
1136-1160\end{array}$ \\
\hline 27 & LE-4592 & $925-927$ & $1089 \pm 40$ & $\begin{array}{l}894-918 \\
954-1008\end{array}$ & 890-1016 \\
\hline 28 & LE-4593 & $930-932$ & $993 \pm 60$ & $\begin{array}{c}996-1058 \\
1080-1122 \\
1134-1160\end{array}$ & $\begin{array}{l}898-910 \\
963-1210\end{array}$ \\
\hline 29 & LE-4594 & $935-937$ & $922 \pm 30$ & $\begin{array}{l}1042-1096 \\
1114-1144 \\
1154-1164\end{array}$ & $\begin{array}{l}1030-1177, \\
1192-1208\end{array}$ \\
\hline 30 & LE-4595 & $938-940$ & $1045 \pm 30$ & 986-1018 & $\begin{array}{l}900-908 \\
966-1030\end{array}$ \\
\hline \multirow[t]{2}{*}{31} & LE-4596 & $942-945$ & $1126 \pm 40$ & $892-968$ & $\begin{array}{l}818-840 \\
858-1012\end{array}$ \\
\hline & \multicolumn{5}{|c|}{ TC-Y111-26-88 (Block V) } \\
\hline \multirow[t]{2}{*}{32} & LE-4791 & $873-960$ & $1143 \pm 30$ & $\begin{array}{l}\text { 886-896, } \\
912-963\end{array}$ & $\begin{array}{l}820-840 \\
860-986\end{array}$ \\
\hline & \multicolumn{5}{|c|}{ TC-Y111-27-130 (Block VI) } \\
\hline 33 & LE-4793 & 879-913 & $1157 \pm 30$ & $\begin{array}{l}\text { 878-898, } \\
910-963\end{array}$ & $\begin{array}{l}792-804 \\
814-848 \\
851-974\end{array}$ \\
\hline 34 & LE-4792 & 914-958 & $1116 \pm 30$ & $\begin{array}{l}892-922, \\
941-976\end{array}$ & 886-991 \\
\hline \multirow[t]{2}{*}{35} & LE-4794 & 879-958 & $1136 \pm 40$ & $\begin{array}{l}886-902 \\
904-966\end{array}$ & $\begin{array}{l}794-802, \\
814-846, \\
853-1002\end{array}$ \\
\hline & $T C-Y 111-2$ & 3-50 (Block & & & \\
\hline 36 & LE-3627 & 971-976 & $1070 \pm 30$ & $970-1014$ & $\begin{array}{l}892-920 \\
946-1022\end{array}$ \\
\hline 37 & LE-3628 & $981-983$ & $1092 \pm 45$ & $\begin{array}{l}892-920 \\
950-1008\end{array}$ & $884-1020$ \\
\hline 38 & LE-3629 & 988-990 & $1113 \pm 40$ & $\begin{array}{l}892-924 \\
936-980\end{array}$ & 876-1016 \\
\hline 39 & LE-3620 & 995-999 & $1045 \pm 30$ & $986-1018$ & $\begin{array}{l}900-908, \\
966-1030\end{array}$ \\
\hline
\end{tabular}


TABLE 2. Radiocarbon Dates of the Novgorod Region and Staraya Ladoga

\begin{tabular}{|c|c|c|c|c|c|c|}
\hline \multirow[b]{2}{*}{ No. } & \multirow[b]{2}{*}{ Lab no. } & \multirow[b]{2}{*}{ Provenience } & \multirow[b]{2}{*}{ Material } & \multirow{2}{*}{$\begin{array}{c}\text { Uncalibrated } \\
\text { date (BP) }\end{array}$} & \multicolumn{2}{|c|}{ Calibrated date (van der Plicht 1993) } \\
\hline & & & & & $1 \sigma, \mathrm{cal} \mathrm{AD/BC}$ & $2 \sigma, \mathrm{cal} \mathrm{AD/BC}$ \\
\hline \multicolumn{7}{|c|}{ Rurikovo Gorodishche 1987-1989 Excavation } \\
\hline \multicolumn{7}{|c|}{ Moat } \\
\hline 1 & LE-3467 & Sq. 237 , depth $3.75 \mathrm{~m}$ & Charcoal & $1340 \pm 80$ & $634-782$ & $\begin{array}{l}558-576 \\
594-884\end{array}$ \\
\hline 2 & LE-3469 & Sq. 238 , depth $5.35 \mathrm{~m}$ & Charcoal & $1240 \pm 50$ & $\begin{array}{l}718-740 \\
768-876\end{array}$ & $\begin{array}{l}672-892, \\
924-934\end{array}$ \\
\hline 3 & LE-3468 & $\begin{array}{l}\text { Sq. } 237 \text {, depth } 4.2-4.6 \\
\text { m }\end{array}$ & Charcoal & $1210 \pm 40$ & $786-878$ & $\begin{array}{l}708-748, \\
762-894, \\
914-957\end{array}$ \\
\hline 4 & LE-3477 & $\begin{array}{l}\text { Sq. 195, pit, depth } 3.7- \\
3.8 \mathrm{~m}\end{array}$ & Charcoal & $1210 \pm 40$ & $786-878$ & $\begin{array}{l}708-748, \\
762-894, \\
914-957\end{array}$ \\
\hline 5 & LE-3476 & Sq. 241 , depth $3.56 \mathrm{~m}$ & Charcoal & $1100 \pm 40$ & $\begin{array}{l}892-920 \\
946-998\end{array}$ & $886-1014$ \\
\hline 6 & LE-3475 & $\begin{array}{l}\text { Sq. 233, pit, depth } 2.18 \\
\mathrm{~m}\end{array}$ & Charcoal & $1020 \pm 100$ & $\begin{array}{l}890-920 \\
952-1160\end{array}$ & $790-1230$ \\
\hline 7 & LE-4404 & $\begin{array}{l}\text { Sq. } 184 \text {, layers on moat } \\
\text { walls }\end{array}$ & Charcoal & $1160 \pm 20$ & $\begin{array}{l}882-893 \\
919-951\end{array}$ & $\begin{array}{l}821-839 \\
865-902 \\
904-967\end{array}$ \\
\hline & \multicolumn{6}{|c|}{ Cultural layer } \\
\hline 8 & LE-3332 & Sq. 201, high layer & Charcoal & $2870 \pm 40$ & $\begin{array}{l}1114-1094 \mathrm{BC}, \\
1072-986 \mathrm{BC}, \\
960-938 \mathrm{BC}\end{array}$ & $\begin{array}{l}1158-1148 \text { вС, } \\
1126-916 \text { вС }\end{array}$ \\
\hline & \multicolumn{6}{|c|}{ "Northern" complex } \\
\hline 9 & LE-4405 & $\begin{array}{l}\text { Sq. } 165 \text {, base of fill: log } \\
\text { on step }\end{array}$ & Wood & $740 \pm 40$ & $1257-1296$ & $\begin{array}{l}1225-1304 \\
1362-1378\end{array}$ \\
\hline 10 & LE-4406 & Sq. 165 , crude boards & Charcoal & $530 \pm 30$ & $1406-1430$ & $\begin{array}{l}1322-1334 \\
1396-1440\end{array}$ \\
\hline 11 & LE-4407 & Sq. $164-171$, base & Wood & $465 \pm 20$ & $1443-1446$ & $1422-1459$ \\
\hline 12 & LE-4408(a) & Posthole in NW & Charcoal & $460 \pm 75$ & $\begin{array}{l}1406-1508 \\
1600-1618\end{array}$ & $\begin{array}{l}1318-1342 \\
1394-1638\end{array}$ \\
\hline & \multicolumn{6}{|c|}{ "Southern" complex } \\
\hline 13 & LE-4411 & Sq. 179 , base & Wood & $770 \pm 50$ & $1236-1287$ & $1170-1300$ \\
\hline 14 & LE-4412 & Sq. 185 , base & Charcoal & $440 \pm 40$ & $1434-1476$ & $\begin{array}{l}1414-1516 \\
1588-1624\end{array}$ \\
\hline 15 & LE-4413 & $\begin{array}{l}\text { Sq. } 175 \text {, black basal } \\
\text { layer }\end{array}$ & Charcoal & $430 \pm 25$ & $1442-1466$ & $\begin{array}{l}1434-1486 \\
1606-1610\end{array}$ \\
\hline 16 & LE-4414 & Sq. 175,182 , base & Charcoal & $450 \pm 30$ & $1434-1463$ & $1420-1482$ \\
\hline 17 & LE-3935 & $\begin{array}{l}\text { Sq. 169-176, gray } \\
\text { humic layer }\end{array}$ & Charcoal & $740 \pm 50$ & $1242-1298$ & $\begin{array}{l}1216-1312 \\
1350-1390\end{array}$ \\
\hline & \multicolumn{6}{|c|}{ Lime-firing kiln } \\
\hline 18 & LE-3333 & $\begin{array}{l}\text { Fill of kiln; depth } 2.14 \\
\text { m }\end{array}$ & Charcoal & $850 \pm 80$ & $\begin{array}{l}1056-1080 \\
1122-1134 \\
1160-1277\end{array}$ & $1032-1287$ \\
\hline 19 & $\begin{array}{l}\text { Valilyevskoe } \\
\text { LE-4157 }\end{array}$ & $\begin{array}{l}\text { 1986-1989 Excavation } \\
\text { Hearth frame (excav. } \\
\text { 1986) }\end{array}$ & Charcoal & $1090 \pm 30$ & $\begin{array}{l}\text { 896-914, } \\
959-1004\end{array}$ & $\begin{array}{l}892-926 \\
932-926\end{array}$ \\
\hline
\end{tabular}


TABLE 2. (Continued)

\begin{tabular}{|c|c|c|c|c|c|c|}
\hline \multirow[b]{2}{*}{ No. } & \multirow[b]{2}{*}{ Lab no. } & \multirow[b]{2}{*}{ Provenience } & \multirow[b]{2}{*}{ Material } & \multirow{2}{*}{$\begin{array}{l}\text { Uncalibrated } \\
\text { date (BP) }\end{array}$} & \multicolumn{2}{|c|}{ Calibrated date (van der Plicht 1993) } \\
\hline & & & & & $1 \sigma, \mathrm{cal} \mathrm{AD/BC}$ & $2 \sigma, \mathrm{cal} \mathrm{AD/BC}$ \\
\hline 20 & LE-4388 & Sq. 31,46 & Charcoal & $1060 \pm 40$ & $\begin{array}{l}900-906 \\
966-1020\end{array}$ & $\begin{array}{l}\text { 892-926, } \\
932-1028\end{array}$ \\
\hline 21 & LE-4389 & Sq. $41,42,51,52$ & Charcoal & $1060 \pm 40$ & $\begin{array}{l}900-906 \\
966-1020\end{array}$ & $\begin{array}{l}\text { 892-926, } \\
932-1028\end{array}$ \\
\hline 22 & LE-4390 & Sq. 51,54 , top & Charcoal & $1090 \pm 40$ & $\begin{array}{l}\text { 892-918, } \\
954-1008\end{array}$ & $\begin{array}{l}932-1028 \\
888-1014\end{array}$ \\
\hline 23 & LE-4391 & Sq. 51,54 , bottom & Charcoal & $1090 \pm 35$ & $\begin{array}{l}894-916, \\
956-1006\end{array}$ & $\begin{array}{l}890-928 \\
930-1012\end{array}$ \\
\hline 24 & LE-4392 & Sq. 55 & Charcoal & $1090 \pm 25$ & $\begin{array}{l}896-912 \\
963-1000\end{array}$ & $\begin{array}{l}\text { 892-922, } \\
936-1012\end{array}$ \\
\hline 25 & LE-3327 & Sq. 55 & Charcoal & $1080 \pm 40$ & $\begin{array}{l}\text { 894-914, } \\
959-1012\end{array}$ & $\begin{array}{l}890-926 \\
930-1018\end{array}$ \\
\hline 26 & LE-3328 & Sq. 63 & Charcoal & $1050 \pm 30$ & 984-1018 & $\begin{array}{l}898-910 \\
963-1028\end{array}$ \\
\hline 27 & LE-3329 & Sq. $56-61$ & Charcoal & $1090 \pm 55$ & $\begin{array}{l}\text { 892-922, } \\
944-1010\end{array}$ & $\begin{array}{l}794-800, \\
816-844, \\
854-1032\end{array}$ \\
\hline & Gorodisch & Jeorgii, 1988 Excavation & & & & \\
\hline 28 & LE-3460 & Sq. 6,7 & Charcoal & $1070 \pm 40$ & $\begin{array}{l}\text { 898-910, } \\
963-1016\end{array}$ & $\begin{array}{l}892-926 \\
932-1024\end{array}$ \\
\hline 29 & LE-3461 & Sq. 2,3 & Charcoal & $1190 \pm 50$ & $\begin{array}{l}780-892 \\
922-938\end{array}$ & $\begin{array}{l}712-744 \\
764-976\end{array}$ \\
\hline 30 & LE-3934 & $\begin{array}{l}\text { Trench, Sec. ZH, depth } \\
2.5-3.0 \mathrm{~m}\end{array}$ & Charcoal & $1020 \pm 70$ & $\begin{array}{c}898-908, \\
965-1054, \\
1082-1122 \\
1136-1158\end{array}$ & $\begin{array}{l}886-1174, \\
1194-1206\end{array}$ \\
\hline 31 & LE-3935 & $\begin{array}{l}\text { Trench, Sec. A-B, dark- } \\
\text { gray charcoal layer }\end{array}$ & Charcoal & $1050 \pm 50$ & $\begin{array}{l}896-912 \\
961-1028\end{array}$ & $\begin{array}{c}886-1046, \\
1092-1116, \\
1142-1156\end{array}$ \\
\hline 32 & LE-3937 & $\begin{array}{l}\text { Construction in sq. } 34 \text {, } \\
37 \text {, basal charcoal layer }\end{array}$ & Charcoal & $1075 \pm 40$ & $\begin{array}{l}896-912 \\
961-1014\end{array}$ & $\begin{array}{l}890-926 \\
930-1020\end{array}$ \\
\hline 33 & LE-3938 & $\begin{array}{l}\text { Clay-charcoal layer, sq. } \\
31,34\end{array}$ & Charcoal & $1080 \pm 30$ & $\begin{array}{l}898-908 \\
965-1010\end{array}$ & $\begin{array}{l}892-922 \\
938-1018\end{array}$ \\
\hline 34 & LE-3939 & $\begin{array}{l}\text { Pit, sq. } 13,14,18,19 \text {, } \\
\text { top of fill }\end{array}$ & Charcoal & $1010 \pm 60$ & $\begin{array}{c}976-1052 \\
1084-1120 \\
1138-1158\end{array}$ & $\begin{array}{l}892-922 \\
940-1170\end{array}$ \\
\hline 35 & LE-3940 & $\begin{array}{l}\text { Pit, sq. } 13,14,18,19 \\
\text { bottom of fill }\end{array}$ & Charcoal & $1100 \pm 40$ & $\begin{array}{l}892-920 \\
946-998\end{array}$ & 886-1014 \\
\hline 36 & LE-3941 & Pit, sq. 39,40 & Charcoal & $1030 \pm 50$ & $\begin{array}{c}900-906, \\
966-1040, \\
1098-1110 \\
1146-1152\end{array}$ & $\begin{array}{c}892-924, \\
936-1060, \\
1067-1124, \\
1130-1160\end{array}$ \\
\hline 37 & LE-3942 & $\begin{array}{l}\text { Construction in sq. } 24 \text {, } \\
25\end{array}$ & Charcoal & $1080 \pm 40$ & $\begin{array}{l}894-914, \\
959-1012\end{array}$ & $\begin{array}{l}890-926, \\
930-1018\end{array}$ \\
\hline 38 & LE-3943 & Cultural layer in sq. 16 & Charcoal & $1050 \pm 50$ & $\begin{array}{l}896-912 \\
961-1028\end{array}$ & $\begin{array}{c}886-1046, \\
1092-1116, \\
1142-1156\end{array}$ \\
\hline
\end{tabular}


TABLE 2. (Continued)

\begin{tabular}{|c|c|c|c|c|c|c|}
\hline \multirow[b]{2}{*}{ No. } & \multirow[b]{2}{*}{ Lab no. } & \multirow[b]{2}{*}{ Provenience } & \multirow[b]{2}{*}{ Material } & \multirow{2}{*}{$\begin{array}{c}\text { Uncalibrated } \\
\text { date (BP) }\end{array}$} & \multicolumn{2}{|c|}{ Calibrated date (van der Plicht 1993) } \\
\hline & & & & & $1 \sigma, \mathrm{cal} \mathrm{AD/BC}$ & $2 \sigma, \mathrm{cal} \mathrm{AD} / \mathrm{BC}$ \\
\hline 39 & LE-3936 & $\begin{array}{l}\text { Trench, Sec. D, depth } \\
0.1-0.3 \mathrm{~m}\end{array}$ & Charcoal & $2350 \pm 70$ & $\begin{array}{l}752-730 \mathrm{BC}, \\
714-716 \mathrm{BC}, \\
530-360 \mathrm{BC} \\
286-254 \mathrm{BC}\end{array}$ & $\begin{array}{l}762-672 \mathrm{BC} \\
666-628 \mathrm{BC} \\
596-576 \mathrm{BC} \\
558-342 \mathrm{BC}, \\
324-200 \mathrm{BC}\end{array}$ \\
\hline \multicolumn{7}{|c|}{$\begin{array}{l}\text { Staraya Lagoda (Zemlyanoe Gorodishche) } \\
\text { Horizon E3 }\end{array}$} \\
\hline 40 & LE-4158 & $\begin{array}{l}\text { Pillar, external tree } \\
\text { rings }\end{array}$ & Wood & $1250 \pm 60$ & $\begin{array}{l}704-748, \\
760-826, \\
834-872\end{array}$ & $\begin{array}{l}\text { 664-892, } \\
920-945\end{array}$ \\
\hline 41 & LE-4159 & Pillar, inner tree rings & Wood & $1275 \pm 55$ & $\begin{array}{l}672-792, \\
804-814, \\
848-850\end{array}$ & $666-880$ \\
\hline 42 & LE-4795 & $\begin{array}{l}\text { Pillar of construction } \\
\text { N3 (1982) (sample } \\
\text { N105), brown humin } \\
\text { (felling date: AD 776- } \\
\text { 811) }\end{array}$ & $\begin{array}{l}\text { Wood } \\
\text { (oak) }\end{array}$ & $1270 \pm 40$ & $680-786$ & $\begin{array}{l}672-826, \\
834-872\end{array}$ \\
\hline 43 & LE-4416 & $\begin{array}{l}\text { Remains of 3rd layer, } \\
\text { sq. D } 30\end{array}$ & Wood & $1085 \pm 40$ & $\begin{array}{l}\text { 894-916, } \\
956-1010\end{array}$ & $890-1016$ \\
\hline 44 & LE-4417 & $\begin{array}{l}\text { 2nd pavement, sq. D28- } \\
\text { E28 }\end{array}$ & Wood & $1010 \pm 40$ & $\begin{array}{r}988-1049 \\
1093-1112 \\
1144-1152\end{array}$ & $\begin{array}{c}970-1064 \\
1074-1126 \\
1132-1162\end{array}$ \\
\hline 45 & LE-4418 & $\begin{array}{l}\text { Top of pavement, sq. } \\
\text { D28-E28 }\end{array}$ & Wood & $1010 \pm 40$ & $\begin{array}{r}988-1049, \\
1093-1112,\end{array}$ & $\begin{array}{r}970-1064, \\
1074-1126\end{array}$ \\
\hline 46 & LE-4419 & Felling frame, sq. Z30 & Wood & $1020 \pm 35$ & $\begin{array}{r}1144-1152 \\
988-1032\end{array}$ & $\begin{array}{l}1132-1162 \\
902-904, \\
966-1052, \\
1086-1120 \\
1138-1158\end{array}$ \\
\hline
\end{tabular}

\section{REFERENCES}

Arslanov, Ch. A. 1987 Radiocarbon: Geochemistry and Geochronology. Leningrad, Leningrad University: 300 p. (in Russian).

Bassalygo, L. A., Sorokin, A. N., Khoroshev, A. S. 1988 The streets of the Troitsky excavation site (topography, stratigraphy, chronology). In Novgorod and the Novgorod Region: History and Archeology. Abstracts. Novgorod: 63-66 (in Russian).

Chernyk, N. B. 1985a Dendrochronology of Ladoga (excavation in the vicinity of Varyagskaya Street). In V. V. Sedov, ed., Ladoga in the Middle Ages: New Archaeological Discoveries and Investigations. Leningrad, Nauka: 117-122 (in Russian).

1985b Dendrochronology of the earliest horizons of Ladoga (based on excavation results at the Zemlyanoe Gorodishche site). In V. V. Sedov, ed., Ladoga in the
Middle Ages: New Archaeological Discoveries and Investigations. Leningrad, Nauka: 76-80 (in Russian). 1987 Dendrochronological scales of the second half of the first millennium $A D$ through the second millennium AD. In T. Bitvinskas, ed., Temporal and Spatial Variations of Climate and Annual Tree-Rings. Kaunas: 90-99 (in Russian).

1989 Chronology and stratigraphy of the lower layers of Zemlyanoe Gorodishche Staraya Lagoda according to dendrochronological dates. In Chernykh, E. N., Scientific Methods in Archaeology. Moscow, Nauka: 201-214 (in Russian).

Kirpichnikov, A. N. 1985 Ladoga in the Middle Ages (Archaeological research). In V. V. Sedov, ed., Ladoga in the Middle Ages: New Archaeological Discoveries and Investigations. Leningrad, Nauka: 3-26 (in Russian). 
Lebedev, G. S. 1985 The Viking Epoch in Northern Europe. Leningrad, Leningrad University Press: 283 p. (in Russian).

Lesman, Yu. M. 1984 Burial monuments of Novgorod and the Novgorod region (the problem of synchronization). In Gaidukov, P. G. and Janin, V. L., eds., Archaeological Research in the Novgorod Region. Leningrad, Leningrad University Press: 118-153 (in Russian).

1990 Chronology of Novgorod jewelry. In Materials on Novgorod Archaeology, 1988. Moscow: 29-98 (in Russian).

Nosov, E. N. 1990 Ryurik Gorodischche Near Novgorod. Leningrad, Nauka: 214 p. (in Russian).

Orlov, S. N. and Aksenov, M. M. 1961 Early Slavic settlements in the Novgorod region. Novgorodsky Istoricheskiy Sbornik 10: 161-168 (in Russian).

Popov, S. G., Svezhentsev, Yu. S. and Zaitseva, F. I. 1991a Radiocarbon and dendrochronology as systems for absolute dating of Novgorod and the archaeological monuments of the Novgorod region. In Janin, V. L, Nosov, E. N. and Gaidukov, P. G., eds., Materials on the Archaeology of the Novgorod Region, 1990. Moscow: 169-182 (in Russian).

1991b Radiocarbon and the absolute chronology of archaeological monuments of the early Middle Ages in northwest Russia. In Gaigalas, A. I., ed., Geochronological and Isotope-Geochemical Investigations in Quaternary Geology and Archaeology. Vilnius, Vilnius University: 131-143 (in Russian).

1993 Radiocarbon chronology of early medieval archaeological sites in northwestern Russia. In Punning, J.-M., Long, A. and Kra, R. S., eds., Applications of Radiocarbon Dating in Eastern Europe and Siberia. Radiocarbon 35(3): 487-493.

Stuiver, M. 1993 A note on single-year calibration of the radiocarbon time scale, AD 1510-1954. In Stuiver, M., Long, A. and Kra, R. S., eds., Calibration 1993. Radiocarbon 35(1): 67-72.

Stuiver, M. and Pearson, G. M. 1993 High-precision bidecadal calibration of the radiocarbon time scale, $A D$ 1950-500 BC and 2500-6000 BC. In Stuiver, M., Long, A. and Kra, R. S., eds., Calibration 1993. Radiocarbon 35(1): 1-23.

Urieva, A. F. 1989 Dendrochronology of planking from the Troitsky excavation in Novgorod. In Chernykh, E. N., Scientific Methods in Archaeology. Moscow, Nauka: 214-228 (in Russian).

van der Plicht, J. 1993 The Groningen Radiocarbon Calibration Program. In Stuiver, M., Long, A. and Kra, R. S., eds., Calibration 1993. Radiocarbon 35(1): 231237. 